\title{
A Mathematical Model of an Evaporative Cooling Pad Using Sintered Nigerian Clay
}

\author{
Chukwuneke Jeremiah Lekwuwa, Ajike Chinagorom Ogbu, Achebe Chinonso Hubert, \\ Okolie Paul Chukwulozie \\ Department of Mechanical Engineering, Nnamdi Azikiwe University, PMB 5025, Awka, Nigeria \\ Email: jl.chukwuneke@unizik.edu.ng
}

Received July 13, 2012; revised August 17, 2012; accepted September 3, 2012

\begin{abstract}
Overtime, reduction in the amount of heat generated in engineering systems in operations have been of great concern and have continuously been under study. It is in line with the above that this research work developed a mathematical model of an evaporative cooling pad using sintered Nigerian clay. A physical model of the evaporative cooling phenomenon was developed followed by the derivation of the governing equations describing the energy and mass transfer for the clay model from the laws of conservation of continuum mechanics. A set of reasonable and appropriate assumptions were imposed upon the physical model. Constitutive relationships were also developed for further analysis of the developed equations. The finite element model of numerical methods was used to analyse the energy transfer governing equations which resulted in the determination of the temperature of the exposed boundary surface at any given time, $t_{2}$ after the commencement of the evaporative cooling processes. In this paper, it was found out that surface temperature differences could be as much as $6^{\circ} \mathrm{C}$ in the first cycle of evaporative cooling with the potential of further reduction. Further, an equation for the prediction of the effectiveness of an evaporative cooling system using clay modeled cooling pads was developed. The findings in this research work can be applied in the design, construction and maintenance of evaporative coolers used to dissipate waste heat when a large amount of natural water is not readily available or if for environmental and safety reasons the large water body can no longer absorb waste heat.
\end{abstract}

Keywords: Evaporative Cooling; Nigerian Clay; Finite Difference Model; Energy Transfer; Cooling Pad; Moulded Clay Material; Heat Transfer; Refrigeration; Temperature

\section{Introduction}

It is general knowledge that from ancient practice, moulded clay materials keep the contents cold. This research work sought to identify to what extent it does this under specified conditions and the scientific proof to that effect. The work went further to do an in-depth analysis of evaporative cooling on sintered clay sample materials and the information obtained by experiment was used to model an evaporative cooling pad.

Evaporative cooling is deemed to be an appropriate alternative cooling mechanism for the cooling of engineering systems in operation due to its simplicity, power saving characteristics as well as its attendant success as a cooling mechanism in other relevant applications. In principle there are two types of evaporative air cooling systems [1-4].

- Direct Evaporative Cooling (DEC)

- Indirect Evaporative Cooling (IEC)

In a Direct Evaporative Air Cooling (DEC) system, air is taken in through porous wetted media or through a spray of water. In the process sensible heat of air evapo- rates some water. The heat and mass transferred between the air and water lowers the dry-bulb temperature of air and increases the humidity at a constant wet-bulb temperature. The dry-bulb temperature of the nearly saturated air approaches the ambient air wet-bulb temperature. The process is an adiabatic saturation one. The wet bulb temperature of the entering airstream limits direct evaporative cooling. This is so because the Dry Bulb Temperature (DBT) of the outgoing airstream can at most be brought to the Wet Bulb Temperature (WBT) of the incoming airstream.

In Indirect Evaporative Air Cooling (IEC) heat transfer between primary and secondary airstreams takes place. The air supplied from outside air to the conditioned space is termed as primary air. The primary air is cooled by secondary air with the help of heat transfer. Secondary air evaporates some of the water which reduces the temperature of secondary air and water. Theoretically temperature of secondary air and water can be reduced to the secondary air wet bulb temperature. Heat transfer takes place from the primary air to the secondary air through 
the wall of the heat exchanger. While constant wet bulb temperature cooling takes place in the path of secondary air and sensible cooling takes place in the path of primary air.

Evaporative cooling of liquid water occurs when the surface of a body of water or moist object is exposed to an open environment which is commonly air. Under these conditions the water will begin to evaporate. The cause of this is explained by $[5,6]$, to be the natural tendency of liquid water seeking to achieve phase equilibrium with the moisture content of the surrounding environment.

As water evaporates, the latent heat of the vaporized water, or heat of vaporization, is absorbed from the body and the surrounding environment. In the absence of other mechanisms of heat transfer (i.e., convection and radiation), a net cooling effect of the body's surface is experienced. In tropical climates, the air in the atmosphere is usually very hot, dry and with a very low relative humidity. Rather than pass air through a refrigerated cooling section, which is quite expensive, it is very much possible to take advantage of the low relative humidity to achieve cooling. This is accomplished by passing air through a water-spray section of the water to be cooled.

Owing to the low relative humidity, part of the liquid - water stream evaporates. The water is cooled simply because of the energy provided by the airstream. The overall effect is a cooling and humidification of the airstream and the process is called Evaporative Cooling, [6]. Evaporative cooling is comparably less expensive and specially suited for climates where the air is hot and humidity is low. It is very important to note that evaporative cooling differs from air conditioning by refrigeration and absorptive refrigeration which use vapour-compression or absorption refrigeration cycles.

\section{Methodology}

In this section, an attempt was made to highlight in clear terms the procedure adopted in accomplishing the set objectives of this research work. They are stated thus:

- Derivation of the governing differential equations describing the energy and mass transfer for the simplified model from the conservation laws of continuum mechanics;

- Simplifying the governing equations through reasonable and appropriate assumptions and the development of constitutive relationships;

- Development of an explicit equation that describes the relationship between the convective heat transfer coefficient hc and mass transfer coefficient $\mathrm{hm}$ as applied to the evaporative system;

- Seeking numerical methods solutions to the resulting equations by the finite element/difference method approach on application of the initial and boundary conditions; and
- Presentation and discussions of the findings of the research work.

\section{Development of Governing Equations}

\subsection{Physical Model}

To adequately examine and study the potential of evaporative cooling in the selected sintered clay material, it was first necessary to develop a physical model of the phenomena and the corresponding governing equations associated with that model on application of relevant physical laws and principles. In the interest of computational time it was convenient to develop a model which was as simple as possible yet complex enough to accurately assess the potential of evaporative cooling for the selected clay sample. Since subsequent experimental research may also be performed to assess the feasibility of the present study it was also important to produce a model whose predictions may be compared with experimental data obtainable from the clay sample material.

The model chosen for the present study is a flat sample of clay material of dimensions $\left(L_{P} \times L_{P} \times L\right)$. In analysis of this nature, heat conduction and mass transfer are inevitable. They occur intermittently due to the temperature and concentration gradient experienced between the water in the sample and its environs. The heat transfer process involves latent heat transfer owing to vaporization of a small portion of the water and the sensible heat transfer owing to the difference in temperature of water and air. The heat and mass transfer account for the cooling of the water in the sample below the inlet temperature $[7,8]$.

The clay material fired to a temperature range of $1000^{\circ} \mathrm{C}$ possesses a measurable level of porosity for evaporation of water. The level of porosity at different temperatures is highest for this sample with a value of $24.72 \%$. This sample is also widely and generally known as earthenware and was used as the model for the analysis in this research work.

An illustration of this model is presented in Figure 1 and Figure 2. The sample material plate possesses a uniform initial temperature and molecular moisture content. At a given time, $t=0$, the surface of the plate is exposed to streams of air containing known free stream temperatures (ambient temperature) [9] and moisture contents (ambient humidity). As a result, heat and moisture will begin to transfer through the plate.

\subsection{Assumptions}

In order to significantly succeed in modelling the sample material in line with the objectives of the work, it was necessary to impose a set of reasonable and appropriate assumptions upon the physical model. This was to simplify the mathematical equations that were formulated in 


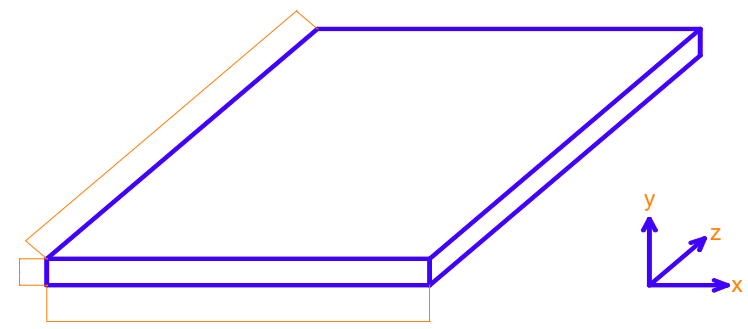

Figure 1. A model of thin flat plate (where $L<<<L_{P}$ ).

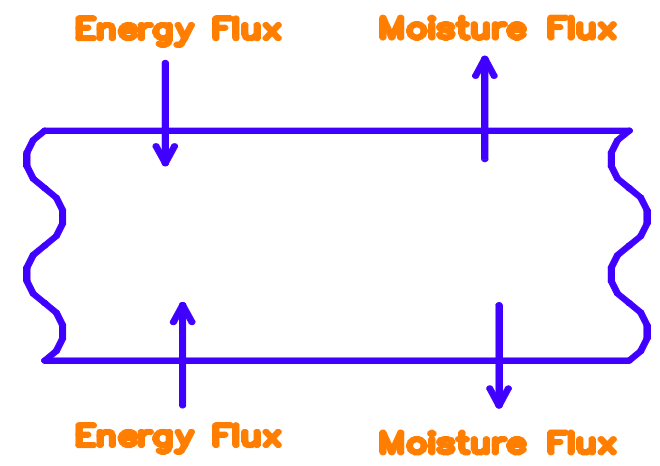

Figure 2. Directions of Heat and moisture transport in the clay sample material.

the course of the work. The overall effect of these as sumptions on the behaviour of the sample clay material was considered to be negligible in the analysis. The assumptions were as follows:

- The moisture bearing clay sample material is isotropic, (i.e. having the same physical properties in all directions). This allowed for the use of Fourier's law of heat conduction;

- The convective heat and mass transfer coefficients on both sides of the plate are uniform. Thus at any location along the y-axis within the plate and for all values of time, $t$, the temperature and molecular moisture content will be uniform in the $x$ and $z$ directions (i.e., independent of $x$ or $z$ ). This reduced the governing equations to one dimensional form;

- Heat transfer at the plate surfaces due to radiation is negligible [7,10]. Diffusion of molecular moisture within the clay sample plate may then be conveniently described by Fick's Law of Diffusion;

- The rate of evaporation is small and does not signifycantly affect the boundary layer of the air flowing over the plate. As a result the convective heat and mass transfer coefficients are considered to be independent of the rate of mass transfer;

- The temperature of the free stream air is less than $100^{\circ} \mathrm{C}$, such that the moisture content of the sample does not boil;

- Evaporation is assumed to occur at the plate surface and not within the plate;

- The thickness of the plate (y-direction) is unaffected by changes in molecular moisture content; and

- Surface tension, stress tensor components as well as capillary forces are to a large extent considered negligible (i.e., zero).

With the preceding physical model and seemingly imposed assumptions from above, it became very much possible to derive the equations governing the behaviour of the clay sample plate depicted in Figure 1 from the basic laws and principles of Engineering. In formulating the governing equations as it concerns evaporative cooling in the model of the sample material selected, it was necessary to consider two fundamental laws. These are the law of conservation of energy and the law of conservation of mass.

\subsection{Finite Element Analysis of a Heat Conducting Clay Slab}

\subsubsection{Theoretical Formulation}

The geometry of field quantities or continuum may be a problem to close form solution of field functions encountered in engineering and science that appropriate algorithm becomes necessary to obtain optimum solution. It is then equally important to employ the "calculus of variation" principles to obtain optimum continuum field functions whose boundary conditions are specified. About all quasi-harmonic phenomena are represented by either the partial derivatives of the function or by the wellknown Laplace and Poisson equation. In calculus of variation, instead of attempting to locate the maxima/ minima points of one or more variables that extremize quantities called functional $(x)$, the function of the functions that extremizes the functional is found [11-14].

The general equation governing quasi-harmonic and time dependent field functions as [4];

$$
\frac{\partial}{\partial x}\left(k_{x} \frac{\partial \phi}{\partial x}\right)+\frac{\partial}{\partial y}\left(k_{y} \frac{\partial \phi}{\partial y}\right)+\frac{\partial}{\partial z}\left(k_{z} \frac{\partial \phi}{\partial z}\right)+Q-c \frac{\partial \phi}{\partial t}=0 \text { (1) }
$$

While the Euler's theorem presented by [6] states that if the integral

$$
I(\phi)=\iiint f\left(x, y, z, \phi, \frac{\partial \phi}{\partial x}, \frac{\partial \phi}{\partial y}, \frac{\partial \phi}{\partial z}\right) \mathrm{d} x \mathrm{~d} y \mathrm{~d} z
$$

is to be minimized, then the necessary and sufficient condition for the minimum to be reached is that the unknown function $\phi(x, y, z)$ should satisfy the following differential equation;

$$
\begin{aligned}
& \frac{\partial}{\partial x}\left[\frac{\partial f}{\partial(\partial \phi / \partial x)}\right]+\frac{\partial}{\partial y}\left[\frac{\partial f}{\partial(\partial \phi / \partial y)}\right] \\
& +\frac{\partial}{\partial z}\left[\frac{\partial f}{\partial(\partial \phi / \partial z)}\right]-\frac{\partial f}{\partial \phi}=0
\end{aligned}
$$

Within the same region provided that $\phi$ satisfies the 
same boundary condition in both cases. From the ongoing, it can be shown that the equivalent formulation to that of Equation (1) is the requirement that the volume integral given below and taken over the whole region, should be minimized.

$$
\begin{aligned}
\chi=\int & {\left[\frac{1}{2}\left\{k_{x}\left(\frac{\partial \phi}{\partial x}\right)^{2}+k_{y}\left(\frac{\partial \phi}{\partial y}\right)^{2}+k_{z}\left(\frac{\partial \phi}{\partial z}\right)^{2}\right\}\right.} \\
& \left.+\left(Q-c \frac{\partial \phi}{\partial t}\right) \phi\right] d x d y d z
\end{aligned}
$$

Subject to $\phi$ obeying the same boundary condition and however, $\partial \phi / \partial t$ is an invariant. In a typical case of one-dimensional 'heat and mass' flow through an isotropic clay surface subjected to a specific boundary condition and devoid of external force (i.e. rate of heat generation $Q=0$ ), the equivalent functional to be minimized reduces to;

$$
\chi=\int \frac{1}{2}\left[k_{y}\left(\frac{\partial \phi}{\partial y}\right)^{2}-\left(c \frac{\partial \phi}{\partial t}\right) \phi\right] \mathrm{d} y
$$

Assuming no accumulation of matter within the sintered clay then $\chi$ can be relaxed for optimization of heat conduction through the thickness of a clay sample. This assumption would be appropriate in a low temperature process displaying free convention without external heat addition. For the particular case of the steady state heat conduction we may identify the functions, $k_{x}, k_{y}$ and $k_{z}$ as isotropic conductivity coefficients, the function $Q$ as the rate of heat generation, the unknown field function as the temperature $(T)$, and $\partial \phi / \partial t$ is due to accumulation of heat at various locations (provided the co-ordinates coincide with the principal axis of the material). The last term of Equation (3) can be considered as a prescribed function of position only. Hence we may re-write Equation (4) in corresponding heat flow terms as;

$$
\chi=\int \frac{1}{2}\left[k_{y}\left(\frac{\partial T}{\partial y}\right)^{2}-\left(c \frac{\partial T}{\partial t}\right) T\right] \mathrm{d} y
$$

The finite element procedure is implemented further by assuming that for the one-dimensional case, heat exchange is executed in a region defined by a straight line (whose length corresponds to the thickness of the plate $w$ ) discretized into a finite number of line elements described uniquely by two nodal points, while the nodes correspond to equivalent isotherms overlaid in a regular fashion across the entire surface as illustrated in Figure 3 below.

The minimization of the integral (functional) given in Equation (5) gives each element's contribution to the solution of the continuum problem when the assembly system is solved. Consider a typical element of the region identified by the 2-nodes in a local co-ordinate sys- tem $(i, j)$. In general, if within the element;

$$
\{T\}^{\mathrm{e}}=\left\{\begin{array}{c}
T_{i} \\
T_{j}
\end{array}\right\} ; N=\left[N_{i} N_{j}\right]
$$

Hence;

$$
T=[N]\{T\}^{\mathrm{e}}=N_{i} T_{i}+N_{j} T_{j}
$$

Similarly;

$$
\frac{\partial T}{\partial t}=[N]\left\{\frac{\partial T}{\partial t}\right\}^{\mathrm{e}}
$$

Equation (7) is the shape function and $N$ is the interpolating function. It has been shown as in (8), (10), (11) and (12) that;

$$
N_{i}=\frac{y_{j}-y}{y_{j}-y_{i}}, N_{j}=\frac{y-y_{i}}{y_{j}-y_{i}}
$$

With the nodal values of $T$ now defining uniquely and continuously the function throughout the region the "functional" $\chi$ can be minimized with respect to these values. This process is best accomplished by evaluating first the contributions to each differential such as $\partial \chi / \partial T_{i}$ from a typical element, then adding all such contributions and equating to zero. Only the elements adjacent to the node $i$, will contribute to $\partial \chi / \partial T_{i}$ just as only such elements contributed in plane elasticity of the equilibrium equation of such node.

\subsubsection{Formulation of Element Equation}

If the value of $\chi$ associated with an element is designated with $\chi^{e}$ (implying integration limited to the length of the element) then we can write by differentiating Equation (5);

$$
\frac{\partial \chi^{e}}{\partial T_{i}}=\int\left[k_{y} \frac{\partial T}{\partial y} \frac{\partial}{\partial T_{i}}\left(\frac{\partial T}{\partial y}\right)+c[N] \frac{\partial T}{\partial t} \frac{\partial T}{\partial T_{i}}\right] d y
$$

With $T$ given as the "shape function" defined by Equation (5); evaluating the partial derivatives contained in Equation (8) we can write;

$$
\begin{aligned}
& \frac{\partial \chi^{e}}{\partial T_{i}}=\frac{1}{L^{2}} \int_{y_{i}}^{y_{j}} k_{y}\left(T_{i}-T_{j}\right) d y+\int_{y_{i}}^{y_{j}} c[N]\left\{\frac{\partial T}{\partial t}\right\}^{e} N_{i} d \\
& =\frac{k_{y}}{L^{2}}\left(T_{i}-T_{j}\right)+\int_{y_{i}}^{y_{j}}\left(c[N] N_{i}\right) d y\left\{\frac{\partial T}{\partial t}\right\}^{e}
\end{aligned}
$$

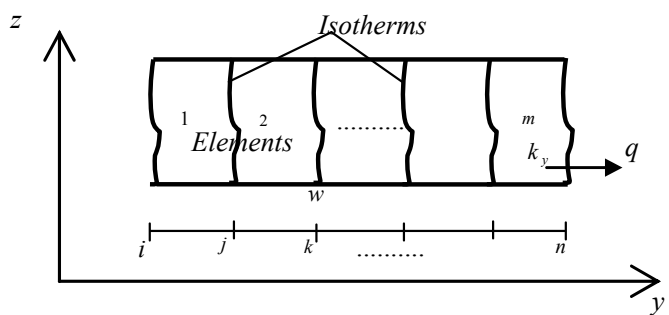

Figure 3. Finite element model of 2-dimensional slab. 
Similarly;

$$
\frac{\partial \chi^{e}}{\partial T_{j}}=\frac{k_{y}}{L^{2}}\left(T_{j}-T_{i}\right)+\int_{y_{i}}^{y_{j}}\left(c[N] N_{j}\right) d y\left\{\frac{\partial T}{\partial t}\right\}^{e}
$$

Combining Equations (11) and (12) gives a typical element equation of the general form $[15,16]$;

$$
\left\{\frac{\partial \chi^{e}}{\partial T}\right\}_{i j}=\frac{k_{y}}{L}\left[\begin{array}{cc}
1 & -1 \\
-1 & 1
\end{array}\right]\left[\begin{array}{l}
T_{i} \\
T_{j}
\end{array}\right]+[p]\left\{\frac{\partial T}{\partial t}\right\}^{e}
$$

where; $L=y_{j}-y_{i}$ is the mesh size.

$$
\begin{aligned}
{[p]=p_{i} j } & =\int_{y_{i}}^{y_{j}} c[N]\left[N^{T}\right] d y \\
& =\frac{2 c}{L^{2}}\left[y_{i}^{2} y_{j}+y_{j}^{2} y_{i}+\frac{1}{3}\left(y_{j}^{3}-y_{i}^{3}\right)\right] I
\end{aligned}
$$

where $I=\left[\begin{array}{ll}1 & 0 \\ 0 & 1\end{array}\right]$ is an identity matrix [15].

Evidently the first product $[N]\left[N^{T}\right]$ is a scalar, hence the value of $[p]$ in computed by multiplying the value of the integral by unit vector to achieve a balanced degree of freedom with the vector $[h]$

Hence, the element equation can be expressed in a more concise form as;

$$
\left\{\frac{\partial \chi}{\partial T}\right\}^{e}=h_{i j} T_{j}+p_{i j} \frac{\partial T_{j}}{\partial t}
$$

\subsubsection{Assembly Equation}

The final equation of minimization procedure requires the assembly of all the differentials of $\chi$ and equating the result to zero. Typically;

$$
\frac{\partial \chi}{\partial T_{i}}=\sum \frac{\partial \chi^{e}}{\partial T_{i}}=0
$$

The summation is taken over all the elements. Hence in the light of Equations (15) and (16) we may write;

$$
\frac{\partial \chi}{\partial T_{i}}=\sum \sum h_{i j} T_{j}+\sum \sum p_{i j} \frac{\partial T_{j}}{\partial t}=0
$$

or;

$$
\frac{\partial \chi}{\partial T_{i}}=[H]\{T\}+[P]\left\{\frac{\partial T}{\partial t}\right\}=0
$$

In which $[H]$ is the 'stiffness matrix' of the whole assembly [9] and $[P]$ is a matrix assembled by precisely the same rule as the stiffness matrix from the components of $p_{i j}$.

\subsubsection{Development/Computation of Final Assembly Equation}

The generation of the final assembly equation for a typical heat conduction surface formulated above may be accomplished in the following synthesized procedures. Suppose we consider a slab of thickness $\mathrm{w}=1 \mathrm{~cm}$, thermal conductivity, $\mathrm{k}=0.72 \mathrm{~W} / \mathrm{m}^{\circ} \mathrm{C}$, specific heat capacity, $\mathrm{C}_{\mathrm{p}}=920 \mathrm{~J} / \mathrm{kg}^{\circ} \mathrm{C}$ and density $\rho=1780 \mathrm{~kg} / \mathrm{m}^{3}$ that is initially at a temperature of $30^{\circ} \mathrm{C}$. Say at time $t=0$, one side of the slab is brought in contact with water at $\mathrm{T}_{\mathrm{w}}=$ $40^{\circ} \mathrm{C}$ at all times, while the other side is subjected to convection to the environment at $\mathrm{T}_{\infty}=30^{\circ} \mathrm{C}$ discretized into five (5) similar elements of length $\mathrm{L}$ composed of a total of six (6) nodal points. The co-ordinates of these points in the universal system may be described as shown in Figure 4. Comparing Equations (13) and (14) shows that $c=\rho C_{p}$ if the accumulation of mass in the slab due to evaporation of water is sufficiently small to guarantee constant density. This assumption is valid in low temperature operation where matter transport is considered negligible.

$p_{i j}$ and $h_{i j}$ are evaluated separately for every individual element in global coordinate system and the assembly follows the method suggested in Section 3.1 obeying consistent element topology and the resulting minimization equation of the global system is expressed in matrix form as follows;

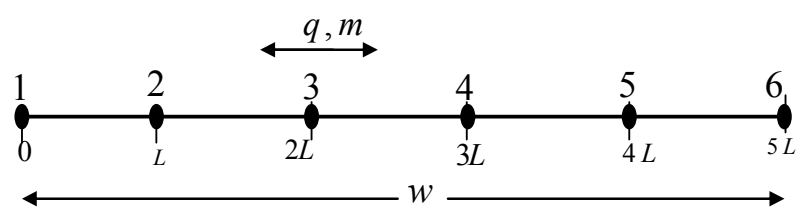

Figure 4. Finite element model of a sintered clay slab subjected to heat exchange with the surrounding.

$$
\frac{k_{y}}{L}\left[\begin{array}{cccccc}
1 & -1 & 0 & 0 & 0 & 0 \\
-1 & 2 & -1 & 0 & 0 & 0 \\
0 & -1 & 2 & -1 & 0 & 0 \\
0 & 0 & -1 & 2 & -1 & 0 \\
0 & 0 & 0 & -1 & 2 & -1 \\
0 & 0 & 0 & 0 & -1 & 1
\end{array}\right]\left[\begin{array}{c}
T_{1} \\
T_{2} \\
T_{3} \\
T_{4} \\
T_{5} \\
T_{6}
\end{array}\right]-c L\left[\begin{array}{cccccc}
2 / 3 & 0 & 0 & 0 & 0 & 0 \\
0 & 4 / 3 & 0 & 0 & 0 & 0 \\
0 & 0 & 4 / 3 & 0 & 0 & 0 \\
0 & 0 & 0 & 4 / 3 & 0 & 0 \\
0 & 0 & 0 & 0 & 4 / 3 & 0 \\
0 & 0 & 0 & 0 & 0 & 2 / 3
\end{array}\right]\left[\begin{array}{c}
\partial T_{1} / \partial t \\
\partial T_{2} / \partial t \\
\partial T_{3} / \partial t \\
\partial T_{4} / \partial t \\
\partial T_{5} / \partial t \\
\partial T_{6} / \partial t \\
\partial t
\end{array}\right]
$$




$$
\left\{\begin{array}{c}
\partial T_{1} / \partial t \\
\partial T_{2} / \partial t \\
\partial T_{3} / \partial t \\
\partial T_{4} / \partial t \\
\partial T_{5} / \partial t \\
\partial T_{6} / \partial t
\end{array}\right\}=\left[\begin{array}{cccccc}
0.15 & -0.15 & 0 & 0 & 0 & 0 \\
-0.075 & 0.15 & -0.075 & 0 & 0 & 0 \\
0 & -0.075 & 0.15 & -0.075 & 0 & 0 \\
0 & 0 & -0.075 & 0.15 & -0.075 & 0 \\
0 & 0 & 0 & -0.075 & 0.15 & -0.075 \\
0 & 0 & 0 & 0 & -0.15 & 0.15
\end{array}\right]\left\{\begin{array}{l}
T_{1} \\
T_{2} \\
T_{3} \\
T_{4} \\
T_{5} \\
T_{6}
\end{array}\right\}_{t}
$$

Equation (20) the global heat diffusion equation from which the time histories of the temperature distribution of the idealized system may be studied if the boundary condition is known or specified.

Substitution of $L=w / 5$ and $c=\rho C_{p}$ and $k_{y}=k$ reduces the assembly equation to the following set of linear first-order equation

\subsubsection{Application of Boundary Conditions/Initial Values}

Equation (20) may not have a definite solution if the values of the objective function (temperature) at the borders and in the beginning of the process are not specified or known. Such specification is usually referred to as Boundary Condition (BC) and initial value respectively. Considering the particular case where the slab is initially at a temperature of $30^{\circ} \mathrm{C}$ (say) at time $t=0$ while one of its ends is brought in contact with water reservoir at $\mathrm{T}_{\mathrm{w}}$ $=40^{\circ} \mathrm{C}$ at all times and the other side is subjected to convection to an environment at $\mathrm{T}_{\infty}=30^{\circ} \mathrm{C}$.

This poses a boundary value problem in $\mathrm{T}(t)$ whose solution would emerge from the substitution;

$$
T_{1}=T_{w}=40, T_{6}=T_{\infty}=30, \partial T_{1} /\left.\partial t\right|_{t}=\partial T_{6} /\left.\partial t\right|_{t}=0
$$

In Equation (20), to obtain the following reduced equation

$$
\begin{aligned}
\partial T_{2} / \partial t & =3-0.075 T_{3} \\
\partial T_{3} / \partial t & =-3+0.15 T_{3}-0.075 T_{4} \\
\partial T_{4} / \partial t & =-0.075 T_{3}+0.15 T_{4}-2.25 \\
\partial T_{5} / \partial t & =-0.075 T_{4}+2.25
\end{aligned}
$$

The resulting set of linear first order differential Equation (19) is now solved subject to

$$
T_{2}(0)=T_{3}(0)=T_{4}(0)=T_{5}(0)=30
$$

as an Initial Value Problem (IVP) with the expediency of
MATLAB "dissolve" command to arrive at the following exponential series;

$$
\begin{aligned}
\left.T_{2}\right|_{t} & =220 / 9+5 / 9 e^{9 / 40 t}+5 e^{3 / 40 t}+1 /\left.4 t_{3}\right|_{t} \\
& =110 / 3-5 / 3 e^{9 / 40 t}-5 e^{3 / 40 t} \\
\left.T_{3}\right|_{t} & =100 / 3+5 / 3 e^{9 / 40 t}-5 e^{3 / 40 t} \\
\left.T_{4}\right|_{t} & =230 / 9-5 / 9 e^{9 / 40 t}+5 e^{3 / 40 t}-1 / 4 t
\end{aligned}
$$

\section{Discussion and Conclusions}

The solution of the interior mesh points presented above with the boundary conditions and the initial values predict completely the time histories of temperature distributionacross the isotherms overlaid through the thickness of the slab (see Figures 5 (a) - (d) below).

The result shows that during the conduction process when " $t$ " probably take value $>0$ the instantaneous nodal temperatures $T_{i}(t)$ assume peak and minimum values between adjacent nodes of a typical element which suffices to say that the diffusion of heat at the nodes closer to heat source for any given element is accompanied by certain degree of heat addition at the adjacent node confirming the quasi-harmonic nature of heat flow across the region while as time approaches infinity the parameter everywhere converges progressively and exponentially to the value fixed at the low temperature reservoir. This as well shows that the excess heat transmitted to the subsurfaces due to the hot interface diffused rapidly into the low temperature reservoir via the oscillation of heat across the thickness of the slab which characterize the conduction process and the associated surface convection, maintaining low temperature zones within the entire region over a long period of time. The heat diffusion process in the sintered clay is partially controlled by its porosity and permeability which allows for interaction of gaseous molecules from the bounding heat reservoirs and the relative heat loss by convection, leading to the socalled evaporative cooling effects. By and large, the low temperature profile maintained over time across the slab 


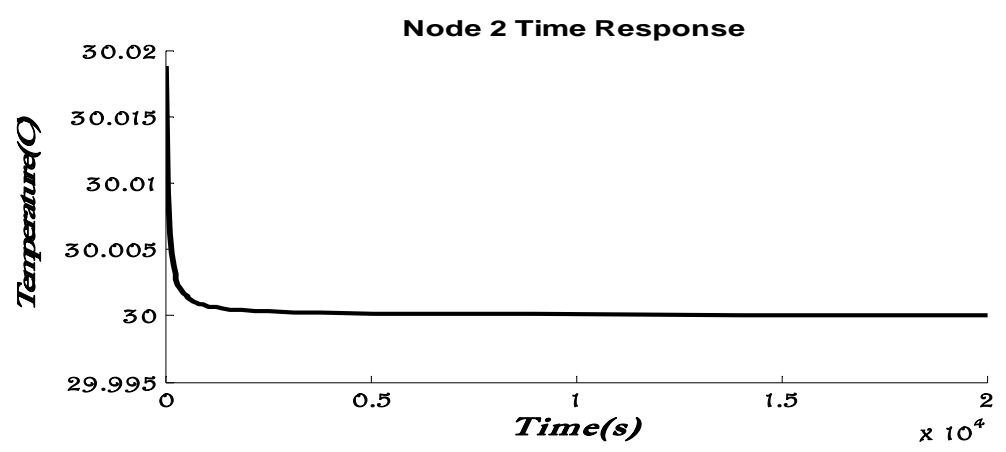

(a)

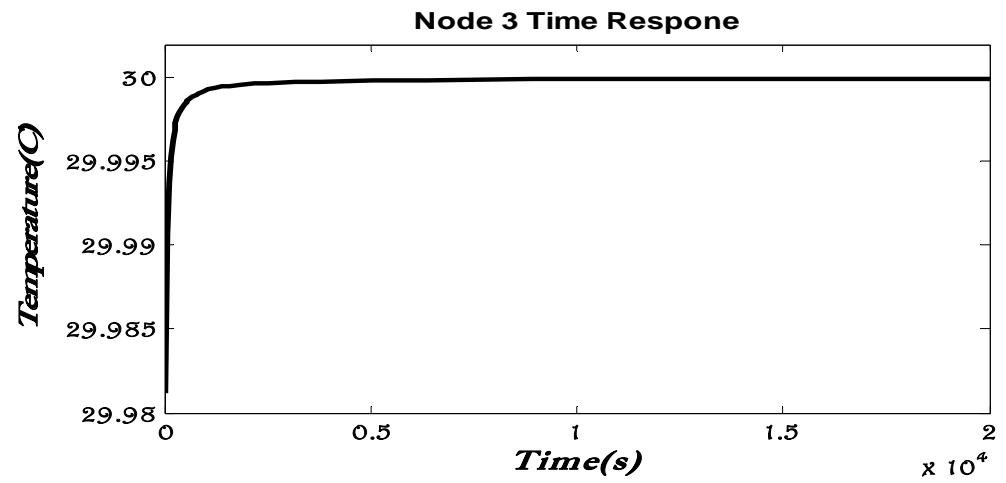

(b)

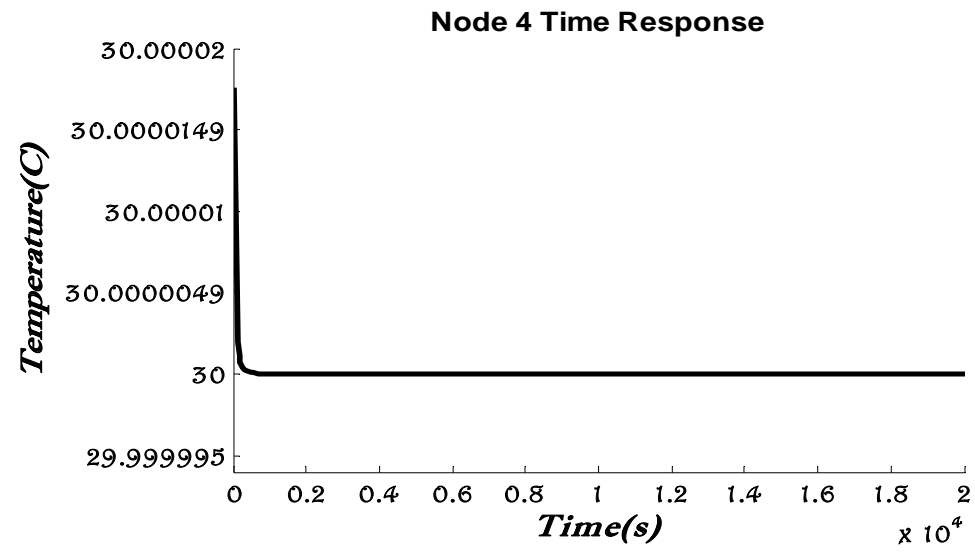

(c)

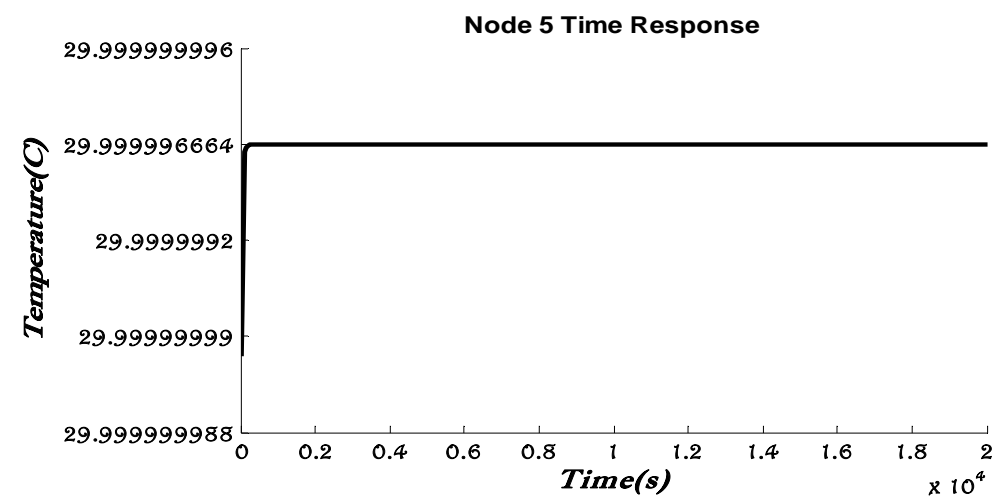

(d)

Figure 5. (a), (b), (c) and (d). The Instantaneous subsurface temperature profile of the field sintered clay slab within the first six (6) hours. 


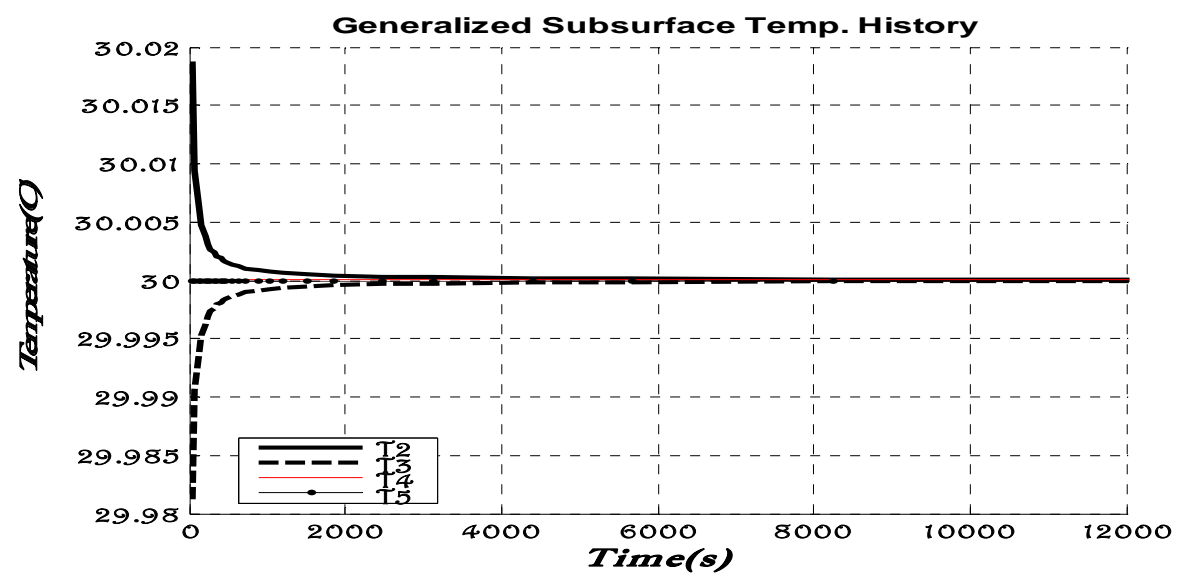

Figure 6. The generalized instantaneous subsurface temperature profile.

can also be attributed to the low thermal conductiveity of the material observed in the model. This result may not however be the same for the case where the size of the heat source or sink (purported reservoir) is not large enough as to sustain constant temperature at the boundaries (creating what may be regarded as a conditioned space). In such conditions, significant cooling effect is identified in space due to removal of sensible heat from the conditioned space in the form illustrated by Figure 5 and Figure 6 but only to normalize on attaining the wet bulb temperature of the surrounding medium. Thisobservation justifies the evaporative cooling potential of the Afikpo clay sample analysed. Other important application may include thermal insulation in high frequency power transmission line, heat sink in electronic gadgets which may not otherwise operate for long in elevated temperatures and other similar processes.

\section{Acknowledgements}

The authors would like to thank Engr.Prof. M. O. I Nwafor, Director Energy Center, Federal University of Technology, Owerri, Nigeria. and Engr. Dr. J. A. Ujam, Senior Lecturer, Nnamdi Azikiwe University, Awka Nigeria, for their intuitive ideas and fruitful discussions with respect to their contribution.

\section{REFERENCES}

[1] F. Al-Sulaiman, "Evaluation of the Performance of Local Fibers in Evaporative Cooling, Energy Conversion \& Management," Elsevier Science Ltd., Amsterdam, 2002.

[2] C. Liao and K. Chiu, "Wind Tunnel Modeling and the System Performance of Alternative Evaporative Cooling Pads in Taiwan Region, Building and Environment," Elsevier Science Ltd., Amsterdam, 2002.

[3] A. Hasan and K. Sirén, "Performance Investigation of Plain and Finned Tube Evaporatively Cooled Heat Exchangers, Applied Thermal Engineering," Elsevier Sci- ence Ltd., Amsterdam, 2003.

[4] Y. J. Dai and K. Sumathy, "Theoretical Study on a CrossFlow Direct Evaporative Cooler Using Honeycomb Paper as Packing Material, Applied Thermal Engineering," Elsevier Science Ltd., Amsterdam, 2002.

[5] Y. A. Cengel and M. A. Boles, "Thermodynamics: An Engineering Approach," 4th Edition, McGraw-Hill, New York, 2002.

[6] W. Kenneth Jr. and D. E. Richards, "Thermodynamics," 6th Edition, McGraw-Hill Companies Inc., New York, 1999.

[7] J. H. Lienhard, "A Heat Transfer Textbook," 3rd Edition, Phlogiston Press, Cambridge, 2005.

[8] R. S. Khurmiand and J. K. Gupta, "Refrigeration and Airconditioning," 3rd Edition, Eurasia publishers, New Delhi, 2004.

[9] E. E. Nnuka and C. Enejor, "Characterization of Nahuta Clay for Industrial and Commercial Applications," Nigerian Journal of Engineering and Materials, Vol. 2, No. 3, 2001, pp. 9-12.

[10] F. P. Incropera and D. P. DeWitt, "Fundamentals of Heat and Mass Transfer," 5th Edition, John Wiley \& Sons, Inc., New York, 2002.

[11] M. L. Averill, "Simulation Modeling and Analysis," 4th Edition," McGraw-Hill Inc., New York, 2007.

[12] O. C. Ziennkiewicz and Y. K. Cheung, "The Finite Element in Structures and Continuum Mechanics," McGrawHill Publishing Company Ltd., London, 1967.

[13] R. J. Astley, "Finite Element in Solids and Structures," Chapman and Hall Publishers, London, 1992.

[14] C. C. Ihueze and S. M. Ofochebe, "Finite Design for Hydrodynamic Pressures on Immersed Moving Surfaces," International Journal of Mechanics and Solids Research India Publications, Vol. 6, No. 2, 2001, pp. 115-128.

[15] M. K. Chain, S. R. K. Iyenger and R. K. Chain, "Numerical Method for Scientific and Engineering Computation," 5th Edition, New Age International (P) Limited Publishers, New Delhi, 2009.

[16] C. C. Ike, "Advanced Engineering Analysis," 2nd Edition, De-Adroit Innovation, Enugu, 2004. 